\title{
Psychiatric epidemiology cross-cultural perspective: a review
}

Yekeen A. Aderibigbe and Adityanjee

\section{Eur Arch Psychiatry Clin Neurosci (1995) 246:37-46}

Regarding the second author, he has no first name or initials; he should be quoted simply as Adityanjee (instead of S. K. Adityanjee, as erroneously published). 